\title{
Periodic-Graph Approaches in Crystal Structure Prediction
}

\author{
Vladislav A. Blatov and Davide M. Proserpio
}

\section{1}

\section{Introduction}

The explosive growth in inorganic and organic materials chemistry has seen a great upsurge in the synthesis of crystalline materials with extended framework structures (zeolites, coordination polymers/coordination networks, metal-organic frameworks (MOFs), supramolecular architectures formed by hydrogen bonds and/or halogen bonds, etc.). There is a concomitant interest in simulating such materials and in designing new ones. In this respect, the role of new topological approaches in the modern crystallochemical analysis sharply increases compared to traditional geometrical methods that have been known for almost a century $[1,2]$. As opposed to the geometrical model that represents the crystal structure as a set of points allocated in the space, the topological representation focuses on the main chemical property of crystalline substance - the system of chemical bonds. Since this system can be naturally described by an infinite periodic graph, the periodic-graph approaches compose the theoretical basis of the topological part of modern crystal chemistry. The history of these approaches is rather long, but only in the last two decades they have come into the limelight. Wells [3] was the first who thoroughly studied and classified different kinds of infinite periodic graph (net) and raised the question: what nets are important for crystal chemistry? This key question for successful prediction of possible topological motifs in crystals was being answered in two general ways initiated by Wells' pioneer investigations.

Firstly, mathematical basics for nets were developed in a number of works concerning quotient graph approach [4-10], special types of nets [11,12], topological descriptors [13-16], and other topological properties related to nets, such as tiles and surfaces [17-19]. Secondly, net abundance and taxonomy were intensively explored in Refs. [20-26]. To solve the emerging problems, novel computer algorithms $[5,16,19,27,28]$, program packages $[6,19,27]$, and electronic databases [19, $27,29]$ were developed that allowed to comprehensively analyze the topological motifs through hundred thousands of crystal structures. With these achievements, materials science and crystal chemistry come up to a new level of their development, that is, characterized by deeper integration of mathematical methods, computer 
algorithms, and programs into modeling and interpretation of periodic systems of chemical bonds in crystals on the basis of periodic-graph representation.

The goal of this chapter is to show that the periodic-graph approaches are very fruitful not only to describe the crystal topologies, but become powerful tools to foresee possible topological motifs, to select most stable ones, and to design novel extended architectures. Besides the achievements of this new field of materials science that we may consider as the theoretical background of the so-called reticular chemistry [30], we will analyze the crucial problems that emerged after its rapid development in the last years.

\section{2}

\section{Terminology}

Since the subject of this chapter is not yet familiar to crystal chemists and materials scientists, we start with a brief summary of the specific terminology used. More detailed set of relevant definitions and the nomenclature of periodic graphs were given in Ref. [31].

Graph is a set of vertices (points), on which a topology is given as a set of ordered pairs of the vertices; each pair determines an edge of the graph. In chemistry, the graph vertices and edges correspond to atoms and interatomic bonds, respectively. The concept of topology is often used by chemists too broadly, without putting a strict sense into it [32]. We emphasize that when treating any molecular structure (crystal can be considered as an infinite molecule), "topology" or equivalently "topological structure" means nothing but the set of all interatomic bonds.

Net is a special kind of infinite graph, that is, simple (without loops, multiple, or directed edges) and connected (any pair of vertices in the graph is connected by a chain of edges); vertices of the graph are called nodes of the net. The coordination number or degree of a node is the number of edges incident on the node. A subnet (supernet) of the net $A$ is a net whose sets of nodes and edges are subsets (supersets) of corresponding sets of $A$. Two nets are isomorphic if there is one-to-one mapping between the sets of their nodes and edges. The symmetry of the net is described by an automorphism group that enumerates all possible permutations of nodes resulting to isomorphic nets. Net is $n$-periodic if its automorphism group contains a subgroup being isomorphic to a group composed by $n$ independent translations. Embedding of the net is a method of allocating its nodes in the space. A net embedding has collisions if it contains coinciding nodes and has "crossings" if some edges intersect. Two embeddings of a net that can be deformed into each other are ambient isotopic. Note that at no point in the deformation may edges intersect or have zero length. Clearly ambient isotopy implies isomorphism (but not vice versa). The symmetry of the net embedding can be lower (but not higher) than the symmetry of the net. For instance, the automorphism group of a net corresponding to a low-symmetric polymorph describes the high-symmetric phase as well. Moreover, there are noncrystallographic nets, whose maximal symmetry cannot be described by a crystallographic space group since any embedding of such 


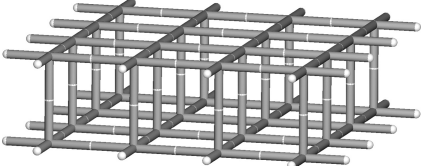

$(4,4)$ la

$\left(4^{8} \cdot 6^{2}\right)-3 \mathrm{D}-2$-periodic

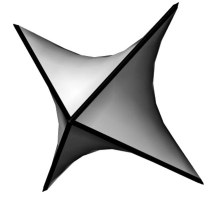

$\left[4^{4}\right]$ tile

(b)

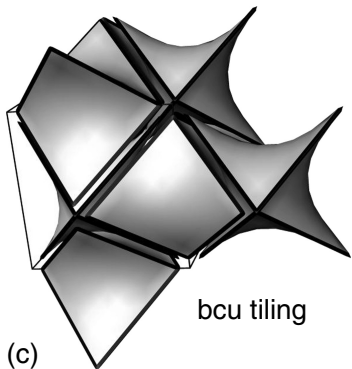

(c)

Figure 1.1 (a) Three-dimensional two-periodic net corresponding to the sphere packing $(4,4)$ la (see [34] for terminology), (b) a single tile, and (c) the tiling for body-centered lattice (bcu). Hereafter we use RCSR bold three-letter symbols [29] to designate the net topology.

net has a lower symmetry. Coordination figure of the node is the solid formed by nodes incident to this node. Dimensionality of the net is equal to the dimensionality of the (Euclidean) space to which the net can be embedded without collisions. We emphasize that the net dimensionality can be larger than the net periodicity; for instance, 3D net can be two-periodic (Figure 1.1). The net is uninodal (bi-, tri-, ..., polynodal) if all its nodes are equivalent (or there are two, three, ..., many inequivalent nodes). If all edges of the net are equivalent, it is called edge-transitive. The net is $n$-regular if the degree of all nodes is equal $n$, even if the nodes are inequivalent. A cycle (circuit) is a closed path beginning and ending at a node, characterized by a size equal to the number of edges in the path (three-circuit, four-circuit, and so on). A ring is an $n$-membered cycle that represents the shortest possible path connecting all the $(n(n-1) / 2)$ pairs of nodes belonging to that circuit. Entangled nets have independent topologies (they have no common edges), but they cannot be separated in any of their embeddings [33]. If the entangled nets have the same dimensionalities and the dimensionality of their array coincides with dimensionality of the separate net, they are called interpenetrating nets. Nodes of one interpenetrating net occupy the cages of the other net, and the edges of one net cross-cycles of the other net. Tile is a generalized polyhedron that can contain vertices of degree 2 and curved faces (that are rings of the net); it corresponds to a topological cage in the net. Tiles form normal (face-to-face) space partition, tiling (Figure 1.1). While any tiling carries a net formed by vertices and edges of tiles, the opposite is in general not true - not any net admits tiling. At the same time, most of nets admit an infinite number of tilings; there is a set of rules [28] to choose a unique, so-called natural tiling. Dual net has nodes, edges, rings, and cages corresponding to cages, rings, edges, and nodes of the initial net; the net and its dual net interpenetrate; if the net is equal to its dual net it is referred to as self-dual. If in this case the vertices of the dual net conform to the cages of the natural tiling, the net is called naturally self-dual. The net with $p$ inequivalent nodes, $q$ inequivalent edges, $r$ inequivalent rings, and $s$ inequivalent cages has the transitivity pqrs; often a shortened symbol $p q$ is used especially if the tiling cannot 
be constructed. The transitivity of the tiling of a dual net corresponds to srpq. Edge net can be constructed by placing new nodes in the middle of the edges of the initial net, connecting new nodes by new edges and removing old nodes and edges. The edge net is complete if all the edges in the initial net are centered by new nodes and all old nodes and edges are removed; otherwise the edge net is partial. Similarly to edge net, one can construct a ring net by putting nodes in the centers of rings of the initial net, removing the nodes of the initial net belonging to the centered rings, and adding new edges between the centers of adjacent rings. Depending on whether all rings are centered or not, the ring net can be complete or partial.

For crystal structure prediction, the most significant nets are one-, two-, and three-periodic, which describe the topology of chain, layer, and framework crystal architectures, respectively. The nets of a higher periodicity as well as nets, whose automorphism group does not contain a subgroup isomorphic to a group of translations, have not yet been explored in relation to chemical objects, albeit they could be useful for aperiodic crystals.

Labeled quotient graph (LQG) of the net is a finite graph whose vertices and edges correspond to infinite sets of translation-equivalent nodes and edges of the net; it describes the net up to isomorphism. In general, LQG can have multiple edges and loops (Figure 1.2). Being finite, it is important for computer storage and processing of nets. The theory of LQGs is intensively being developed by Eon, Klee, and Thimm [7-9].

Topological index of the net is a set of numbers that characterize the net topology. The most rigorous topological index is adjacency matrix, that is, a quadratic matrix

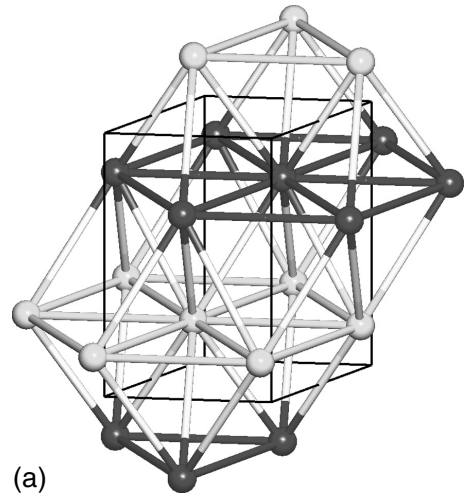

Figure 1.2 (a) A fragment of hcp net (hexagonal close packing) with the translation-equivalent nodes of the same shade of gray. (b) The two nodes in the primitive cell form the vertices of the LQG. (c) The links to the same translation-equivalent nodes (black-to-black, gray-to-gray) correspond to the loops in the LQG, while the links between the two nodes correspond to the sextuple edge of
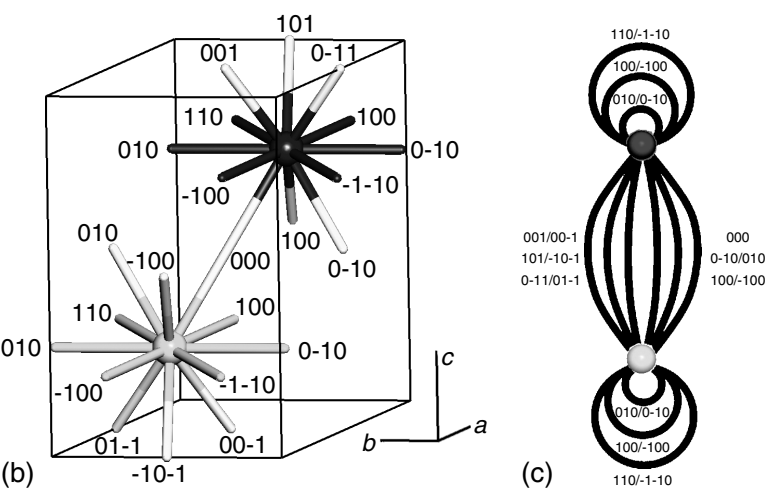

the quotient graph. The three-integer labels uvw show which translation-equivalent nodes are connected with the corresponding node of the net in the primitive cell. Depending on the direction chosen on the LQG, there are always two possible labels uvw or its centrosymmetric $-u-v-w$ : for example, going out of the cell along 100 is equivalent to enter from -100 . 
$M$, whose rows and columns correspond to vertices of the LQG, and each entry $M_{i j}$ shows the existence of edge between $i$ th and $j$ th vertices. The adjacency matrix defines the net and its LQG up to isomorphism. Some other kinds of topological indices are useful to identify and compare net topologies [13-16, 35].

\section{3}

\section{The Types of Periodic Nets Important for Crystal Structure Prediction}

Since there is an infinite number of topologically different periodic nets and an infinite number of geometrically different embeddings of a particular net, it is important to determine the types of nets to be most important for predicting topological features of crystal structures. This work has mainly been done by O'Keeffe and coworkers during the last two decades [21, 36, 37]; it still proceeds now. Three-periodic nets were studied in much more details than low-periodic ones (see Section 1.6); the special types of nets are described below with the example of three-periodic nets.

The easiest to enumerate are the most symmetrical topologies that have the smallest number of inequivalent nodes and/or edges of the net. Hence, uninodal and binodal nets as well as edge-transitive nets are of special interest for crystal chemistry. Among them, one can separate five regular nets: srs, nbo, dia, pcu, and bcu (Figure 1.3), where the coordination figures are regular solids (triangle, square, tetrahedron, octahedron, and cube, respectively), one quasiregular net (fcu) with two kinds of faces in the coordination figure (cuboctahedron), and 14 semiregular nets that are both uninodal and edge-transitive. Of other nets, $n$-regular nets and minimal nets (for the latter holds that removing any set of equivalent edges gives rise to decrease of the net periodicity) are important to crystal structure prediction $[12,37,38]$.

In chemical compounds, the atoms tend to keep neighboring atoms at similar distances; as a rule, the difference in distances for a particular pair of atoms does not exceed several tenths of angstrom. In this respect, sphere packings are most important: they describe net embeddings with all equal edges corresponding to the shortest distances between nodes, only the first neighboring nodes are connected, all other nodes are placed at larger distances. At present, all uninodal sphere packings are known for one- and two-periodic nets [34]; the search for three-periodic uninodal nets is almost finished [39, 40, and references therein]; at least we know all three-periodic uninodal sphere packings that emerge in crystals.

We also mention a special group of self-dual nets, that is, closely related to interpenetrating structures; since the nodes of one net occupy the cages of another net in the interpenetrating array, the net isomorphic to its dual net will be the most suitable to form such an array. At present, 13 naturally self-dual nets are listed in the reticular chemistry structure resource $(R C S R)$ database [29]: cds, dia, ete, ftw, hms, mco, pcu, pyr, qtz-x, sda, srs, tfa, and unj (Figure 1.4). Possible types of interpenetration of homogeneous sphere packings were derived in Ref. [11]; the 


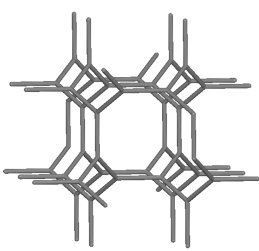

$\left(10^{3}\right)$-srs [1111]

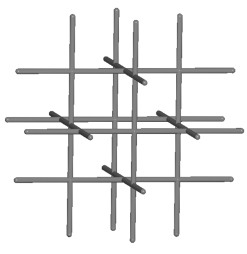

$\left(6^{4} .8^{2}\right)-$ nbo $[1111]$

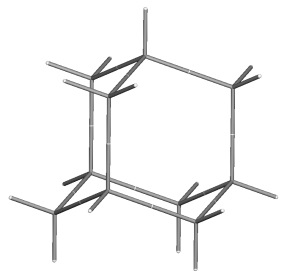

$\left(6^{6}\right)-$ dia [1111]

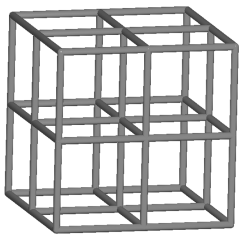

$\left(4^{12} \cdot 6^{3}\right)-$ pcu $[1111]$

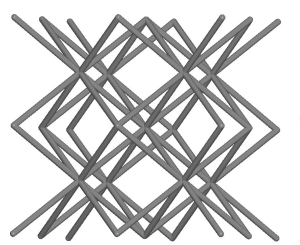

$\left(4^{24} \cdot 6^{4}\right)$-bcu [1111]

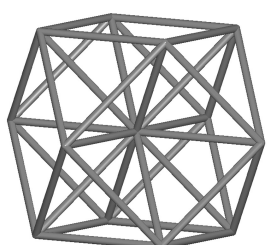

$\left(3^{24} \cdot 4^{36} \cdot 5^{6}\right)-$ fcu [1112]

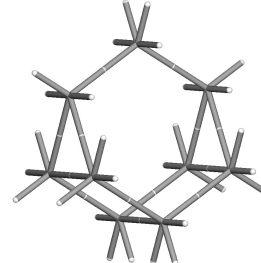

$\left(3^{3} \cdot 4^{6} \cdot 5^{5} \cdot 6\right)-\mathbf{s x d}$ [1331]

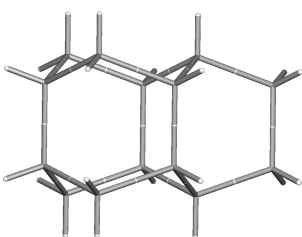

$\left(6^{6}\right)$-Ion [1222]

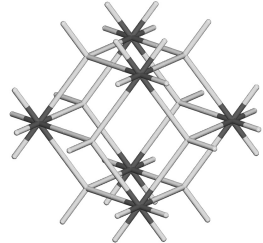

$\left(4^{12} \cdot 6^{12} \cdot 8^{4}\right)\left(4^{6}\right)$-flu [2111]

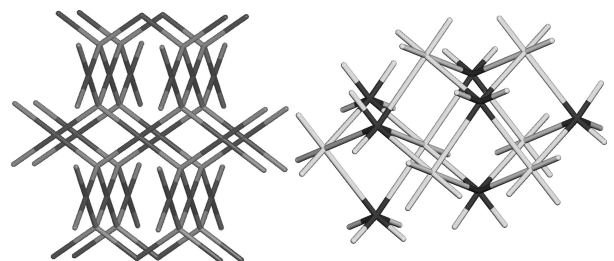

$\left(4^{2} .8^{4}\right)$-pts [2132]

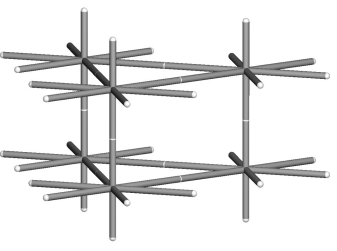

$\left(3^{6} .4^{18} \cdot 5^{6} .6\right)-$ hex $[1221]$

$\left(4^{12} \cdot 6^{3}\right)\left(4^{9} \cdot 6^{6}\right)-$ nia [2122]

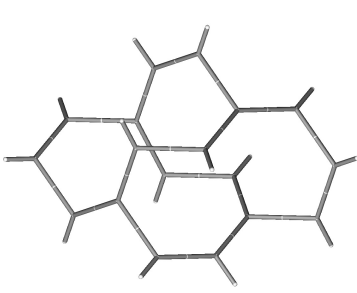

$\left(10^{3}\right)$-utp [1321]

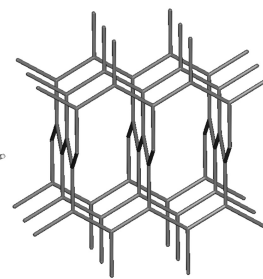

$\left(10^{3}\right)$-ths [1211]

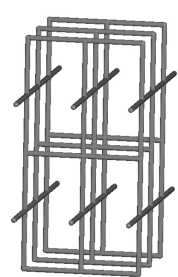

$\left(6^{5} .8\right)-\mathbf{c d s}[1221]$

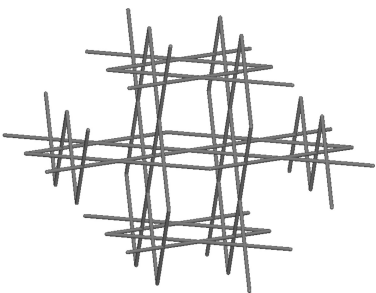

$\left(4^{2} \cdot 8^{4}\right)$-Ivt [1121]

Figure 1.3 List of some important nets. Each net is characterized by its point symbol, RCSR three-letter name and transitivity [29].

classes of interpenetration were considered [20,22] in relation to types of symmetry operations relating the nets in the array.

An efficient way to find crystallochemically important nets and to generate new ones is to search for subnets of known nets. Blatov and Proserpio [16, 41] obtained all uninodal and binodal subnets (totally more than 48000 ) for almost all known nets of the types described above. But not less important is to generate nets $a b$ initio, 


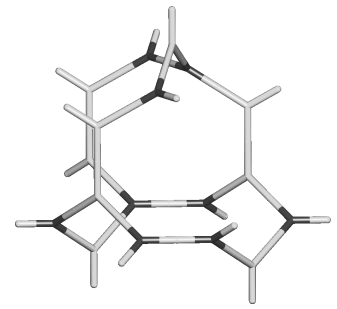

$\left(8^{2} \cdot 10\right)$-ete [2332]

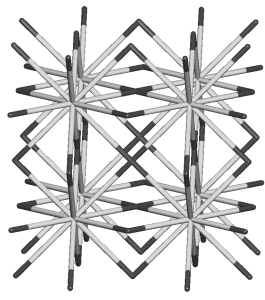

$\left(4^{36} .6^{30}\right)\left(4^{4} \cdot 6^{2}\right)_{3}-\mathrm{ftw}$

[2112]

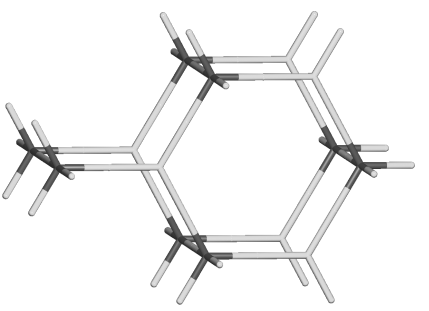

$\left(6^{3}\right)\left(6^{9} .8\right)-\mathrm{hms}$ [2222]

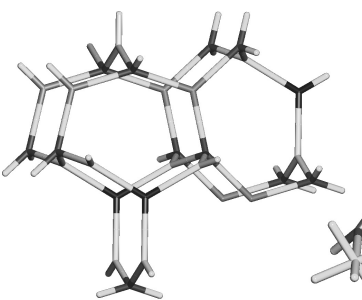

$\left(8^{3}\right)_{2}\left(8^{6}\right)-$ mco [4334]
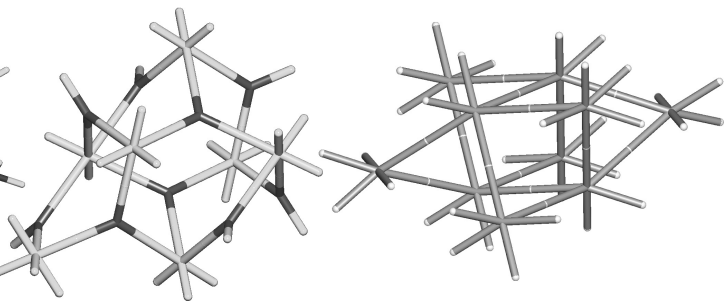

$\left(6^{12} \cdot 8^{3}\right)\left(6^{3}\right)_{2}-\mathrm{pyr}$

[2112]

$\left(4^{4} .5^{10} .7\right)-q t z-x$

[1221]

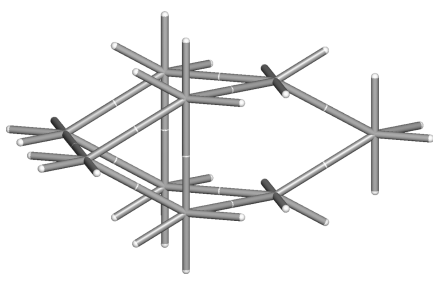

$\left(3^{3} \cdot 5^{9} \cdot 6^{2} \cdot 7\right)-$ sda [1221]

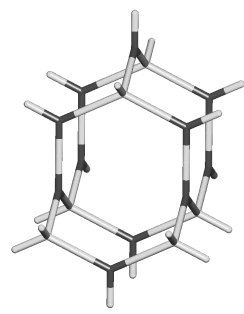

$\left(8^{3}\right)_{2}\left(8^{6}\right)-\mathbf{t f a}$

[2222]

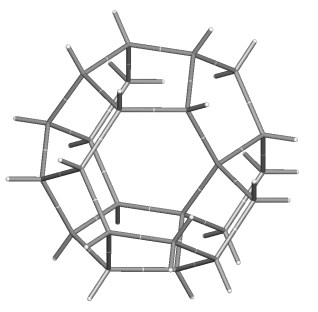

(5 $\left.5^{5} .8\right)$-unj

[1221]

Figure 1.4 Nine self-dual nets. For srs, cds, dia, and pcu see Figure 1.3.

independently of peculiarities of crystal structures. Analyzing such nets, one can see which of their features prevent them from realizing in nature. A very fruitful method was proposed by Hyde and coworkers [19, 42] to obtain three-periodic nets (epinets) by projecting 2D hyperbolic tilings.

\section{4}

\section{The Concept of Topological Crystal Structure Representation}

The question "what net corresponds to a given crystal structure" is not so easy as it likely seems. Any crystal structure can be represented in different ways depending on which interatomic interactions and structure groups we take into account. For instance, we can consider the crystal structure of an ice polymorph as 
a packing of water molecules, as a net of water molecules connected by hydrogen bonds or as a net of oxygen and hydrogen atoms linked by valence and hydrogen bonds. Of course, some other more or less exotic variants can be considered, for example, packing of hydrogens, but usually they are chemically meaningless. How to enumerate all possible topologies and select reasonable ones? Thus, we come to the concept of topological crystal structure representation [43], that is, defined as a net generated from the complete net of the crystal structure by some method of its simplification. By complete net, we mean the net that includes all atoms of the structure as the nodes and all possible, even weakest, atomic interactions as the edges; the complete crystal structure representation corresponds to this net. This hypothetical net describes the structure topology in all details, but in any particular crystallochemical investigation we have to simplify it somehow to obtain a partial representation. The following simplification procedures with the complete net enable us to produce any structure representation: (i) removing some edges; (ii) removing some nodes together with the incident edges; (iii) contracting some nodes to other nodes or to some other points (commonly the molecular centers of mass, the barycenter) keeping the net connectivity.

The first simplification procedure is explicitly or implicitly used in any description of crystal structure. Ordinarily, we consider only strong interactions like valence, ionic, or metallic bonding and ignore van der Waals or even weaker interactions (e.g., Coulomb interactions between long-distant ions). We apply the second simplification procedure when, for instance, interstitial ions or molecules are omitted in zeolites or MOFs. The last simplification procedure corresponds to the representation of polyatomic groups as structureless particles, when we analyze packings of inorganic ions in complex salts or organic molecules in molecular crystals. Most crystal structure representations are the result of a combination of the simplification procedures. For example, one can treat the ice VIII polymorph as an assembly of hydrogen and oxygen atoms connected by valence and hydrogen bonds applying only the first simplification procedure to omit other weak $\mathrm{H} \cdots \mathrm{O}$ and $\mathrm{H} \cdots \mathrm{H}$ interactions, which results in the $\mathrm{SiO}_{2}$-cristobalite topology of the corresponding net. Moreover, there are two such equivalent interpenetrating nets in the structure like in $\mathrm{Cu}_{2} \mathrm{O}$. The next way is to consider the structure as a net of water molecules connected by hydrogen bonds. To provide this representation, one has to contract/remove hydrogen atoms to oxygens, which gives rise to the diamond (dia) topology and, hence, to the array of two interpenetrating dia nets (Figure 1.5). At last, the contraction procedure can be applied with accounting all interactions $\mathrm{H} \cdots \mathrm{O}$ and $\mathrm{H} \cdots \mathrm{H}$ between neighboring molecules to obtain the single net of the body-centered cubic (bcu-x) topology corresponding to molecular packing, where each water molecule contacts 14 other molecules (Figure 1.5). More examples of different crystal structure representations are given in Section 1.7.2.

As a result of simplification, we obtain an underlying net that elucidates the general topological motif of the crystal structure. It follows from the aforesaid that several underlying nets can correspond to the same structure depending on the simplification method. 


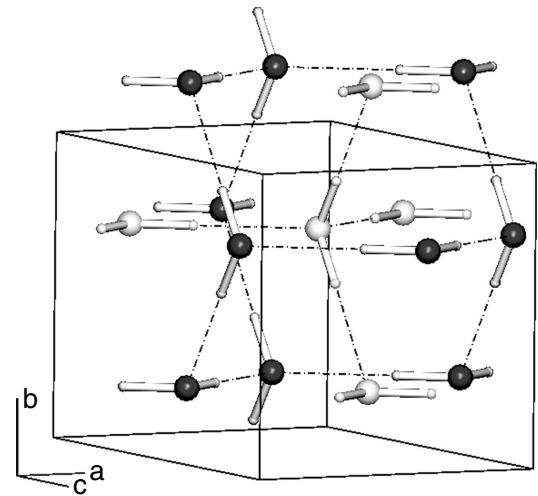

(a)

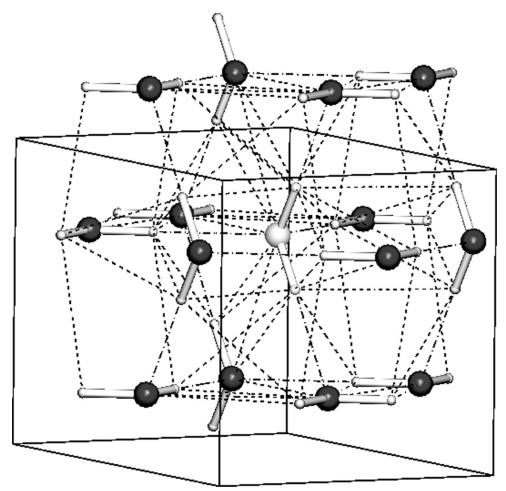

(b)

Figure 1.5 The crystal structure of ice VIII polymorph: (a) as an array of two interpenetrating diamondoid dia nets; (b) as a single bcu-x net; the environment of a water molecule with 14 other molecules is shown. Hydrogen and van der Waals bonds are shown as dash-dotted and dashed lines, respectively.

$\left(6^{6}\right)$-dia [1111]

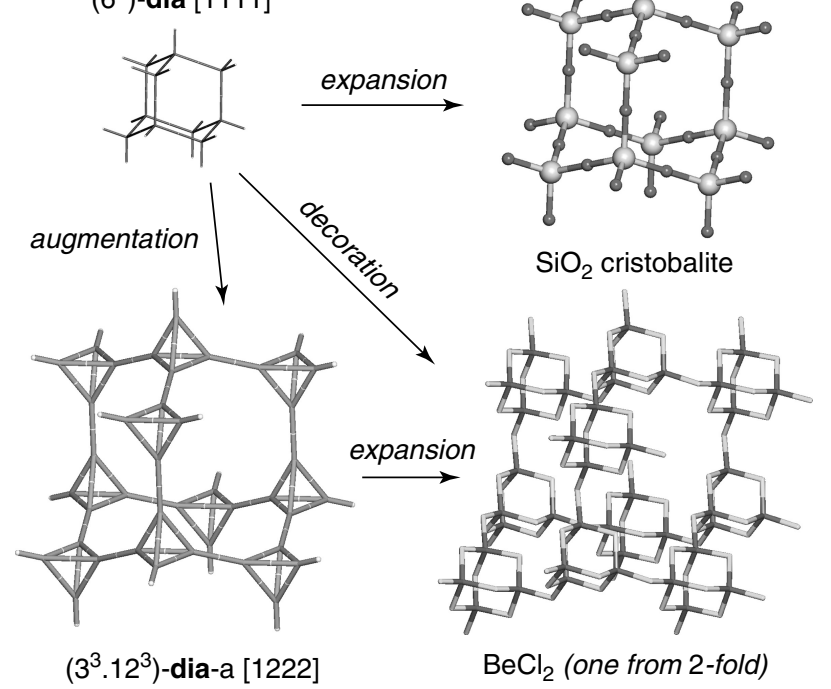

Figure 1.6 Examples of decoration, augmentation, and expansion transformations.

The transformations, being inverse to the simplification procedures, are the decoration of the net nodes (with the special case of augmentation in which the nodes of the original net are replaced by a group of vertices with the shape of the original coordination figure of the node) [29] and the expansion of the net edges; they are the main operations of reticular chemistry [30, 44] (Figure 1.6). 
Thus, the question, what is the single, "true" topology for a particular compound, is often meaningless, but when predicting the crystal structure we have to foresee as many structure representations as possible. From topological point of view, a comprehensive prediction of the crystal structure means finding its complete net since all other representations can be derived from the complete one. In practice, we can predict only some partial representations. For example, we can state that most of molecular packings tend to have topologies of the close packings fcc or hcp; however, we cannot often predict how the atoms of molecules are allocated in the space. Even having a comprehensive X-ray information on the molecule geometry and environment, sometimes we cannot prove if some interactions exist or not. The concept of crystal structure representation allows us to realize which level of the structure understanding we have accomplished and what other representations can be derived from this one.

\section{5}

\section{Computer Tools and Databases}

New tasks emerged in crystal chemistry at the end of the 20th century invoked new tools for their solution. Since the solution should rely on a great deal of crystallographic data, the tools had to provide electronic processing of the information. At that time, just about 10 years ago, there were worldwide electronic databases that accumulated general crystallographic information on almost all studied crystal structures, but these databases were used mainly as electronic handbooks, not as tools to search for crystallochemical regularities or to predict novel crystal architectures. Principally, new software should be developed to automate main stages of crystallochemical analysis and to process the databases in a batch mode. This software naturally uses the periodic net concept to represent and explore the crystal structure. Now we have the following program packages that are distributed free of charge:

Gavrog $^{1)}$ includes two programs: Systre (Symmetry, Structure Recognition, and Refinement) [6] finds the net embedding with maximal symmetry and provides the topological classification using a built-in archive of more than thousand nets taken mostly from the RCSR database (see below); $3 d t$ ( $3 D$ tiler) visualizes three-periodic tilings [37] and computes their topological parameters.

Ole $x^{2)}$ [45] provides, besides some standard procedures of structure determination and analysis, the tools for topological simplification of the initial periodic net and computing a number of its topological indices.

TOPOS $^{3)}$ [27] accumulates all topological approaches mentioned in the previous parts and provides the study of both individual crystal structures and of large groups of structures stored in electronic databases. Almost all comprehensive investigations of net occurrence discussed in Section 1.6 were performed with

1) Generation, Analysis, and Visualization of Reticular Ornaments using Gavrog; http:// www.gavrog.org.
2) http://www.olex2.org.

3) http://www.topos.ssu.samara.ru. 
TOPOS. TOPOS is integrated with databases on topological types TTD and TTO described below.

Using the topological software we can determine a number of crystal structure parameters and perform some operations to be important for the structure classification, rationalization, and prediction: (i) topological type of the underlying net (including the type of interpenetration, if any) and local topology of complex group; (ii) maximum-symmetry embedding for new nets; (iii) automatic search for the net representations; (iv) automatic simplification of the net; (v) search for supernet-subnet relations; (vi) search for a given finite fragment in the net; (vii) performing all the operations both for single crystal structure and for large number of structures via interface with crystallographic databases. The program packages contain tools to discover general tendencies in formation of topological motifs; many hypotheses, or models based on the periodic net concept can now be rapidly and comprehensively checked. Examples will be considered in Sections 1.6 and 1.7.

Gavrog Systre and TOPOS were used to create a new type of electronic databases that can be called crystallochemical since their main information is related to the topology of periodic nets of chemical bonds as opposed to crystallographic databases that are focused on the arrangement of atoms in the space. To create a record in a crystallochemical database, one has to restore a periodic net from the crystallographic data, simplify it if required, reduce the resulting infinite net into an LQG, and store the adjacency matrix of this graph as well as other its topological indices. This can be done only with the tailored software described above. Currently, the following free crystallochemical databases are developed:

$R C S R^{4)}[29]$ is the oldest one, but of age less than 10 years. It contains maximum symmetry embeddings of more than 1700 two- and three-periodic nets considered important for crystal chemistry and crystal design. Most of the nets were found in crystal structures, the remaining ones can be considered as suitable templates for new materials. One of the reasons to decide in favor of a particular crystal structure representation is if its topology is found among the RCSR nets. RCSR uses three-letter symbols to denote the net topologies; these symbols are used in this chapter.

EPINET $^{5)}$ project announced in 2005 [19, 42], strictly speaking, cannot be considered as a crystallochemical database since it collects the nets generated $a b$ initio, irrespective of crystal structures. However, it is strongly important to interpret the results of net occurrence and to develop the methods of net topology prediction as will be shown in Section 1.6. Now EPINET includes 14532 epinets, 162 of them coincide with RCSR nets.

TTD and TTO collections ${ }^{6}$ [41] form an integrated set; the TTD part contains the information on topological indices of almost 68000 topological types of nets including RCSR and EPINET, and the TTO part collects the links between the topological types and the crystal structure data stored in crystallographic databases.

4) Reticular Chemistry Structure Resource, http://rcsr.anu.edu.au/.

5) Euclidean Patterns in Non-Euclidean Tilings, http://epinet.anu.edu.au/.
6) TOPOS Topological Databases and Topological Types Observed, http://www.topos. ssu.samara.ru. 
TOPOS uses this set to determine crystal structure topology and to find other topologically similar compounds.

What information useful for the prediction of topological motifs can we extract from the topological databases? Resting upon the topological type of the net, we can immediately draw some conclusions. (i) If the type is stored in $R C S R$, this topology is not occasional; it was already considered due to some of its important peculiarities. If the net is absent in the databases, there are some special reasons that have invoked the net and we have to discover them. (ii) Maximum symmetry embedding including symmetry of nodes and coordination figures is useful to apply the symmetry criteria discussed in Section 1.7.1. (iii) If the net has some special features (see Section 1.7), the corresponding topological motif could be particularly stable. (iv) Knowing other structures with the same topology, we can find similarities between the compounds and materials of different composition, chemical nature, and structure details that are inessential in the given topological representation. But the most important point is that the tools described in this part allow us to solve currently the crucial problem of crystal chemistry, the nonuniform abundance of periodic nets in nature.

\section{6}

\section{Current Results on Nets Abundance}

The question why some topological motifs frequently occur in nature while other nets have been never found is a crucial point in understanding how the resulting crystal architecture depends on chemical composition and bonding in the substance. Quantum-mechanical technique can hardly be applied as a universal tool to properly answer this question since different topological models of the same structure often lie too close to each other on the energy landscape [46, 47]. Hence, some empirical regularities based on geometrical and topological properties of the structures should be discovered. Being less general than the quantum-mechanical approach, they have the advantage to be drawn and verified with the whole immense array of experimental crystallographic data. The first step in solving this problem is to build statistical distribution of the net topologies over all known periodic structures. This task is very time-consuming, but recent progress in computer methods of the net analysis allows us to hope that it will be finished quite soon. Moreover, even current results on nets occurrence can provide some conclusions to be important for successful topology predictions.

The first extensive investigation of net occurrence was reported in Ref. [20] for interpenetrating valence-bonded 3D architectures. Using TOPOS, the authors processed the whole CSD and obtained a comprehensive list of 301 interpenetrating coordination polymers. It was found that the three most preferred topological motifs are dia, pcu, and srs. Just after a year, O'Keeffe and coworkers [21] published the statistics for 774 valence-bonded single nets in MOFs with the same sequence of the first three leaders. In the next 4 years, the crystal structure topologies in organic, inorganic, and metal-organic compounds were systematically investigated 


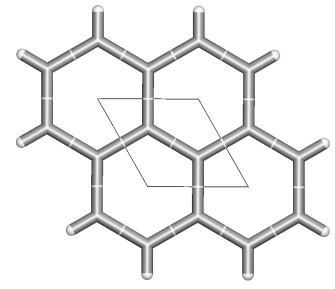

$\left(6^{3}\right)$-hcb [111]

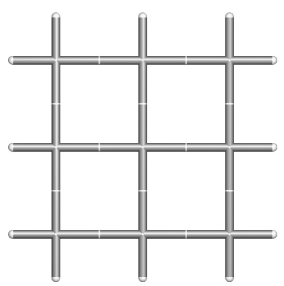

$\left(4^{4}\right)$-sql [111]

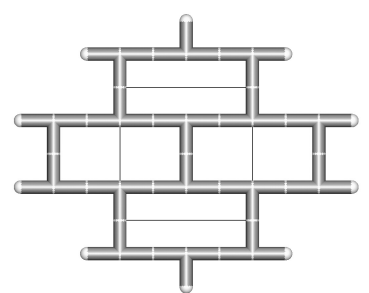

$\left(6^{3}\right)$-hcb [121]

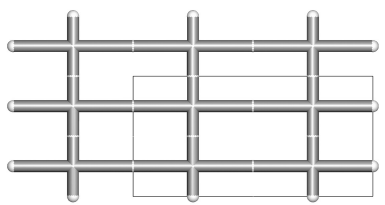

$\left(4^{4}\right)$-sql [121]

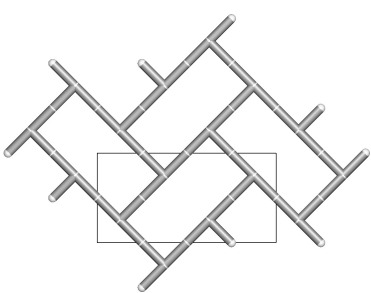

$\left(6^{3}\right)$-hcb [121]

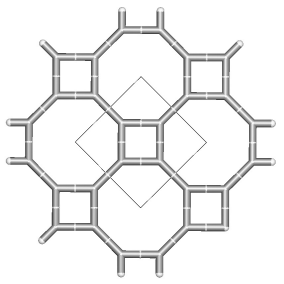

$\left(4.8^{2}\right)$-fes [122]

Figure 1.7 The three most abundant plane nets hcb, sql, fes with some low symmetry embedding (italicized).

[22-26, 48]; most results of these studies are collected in the TTO databases. Thus, in valence-bonded interpenetrated inorganic and MOFs as well as in single MOFs, the leaders are dia-pcu-srs; in organic molecular crystals with hydrogen-bonded single networks, the results are similar (dia-pcu-sxd-hex) as well as for hydrogen-bonded coordination compounds (pcu-bcu-hex-dia).

It seems strange, but all published statistics concern only three-periodic structures of organic, inorganic, and coordination compounds; the low-periodic nets were not explored. At the same time, in two-periodic motifs the preference of some topological types is much sharper than in three-periodic structures. Thus, our analysis of 1711 two-periodic coordination networks shows that the first three places are occupied by sql (37.2\%), hcb (17.3\%), and fes (7.0\%) plane nets (Figure 1.7). Moreover, if we consider only the contribution for the nets of the same coordination, it becomes much more expressed: hcb and fes compose $65.1 \%$ and $26.2 \%$ of three-coordinated nets, respectively, and sql covers $88.6 \%$ of four-coordinated nets. Similar results are obtained for interpenetrating two-periodic arrays. Thus, for 271 coordination networks containing valence-bonded interpenetrating layers, the most abundant layer topologies (84\%) are sql (59.4\%), hcb $(24.0 \%)$, and fes (4.8\%).

As was mentioned above, besides the analysis of crystal structures of chemical compounds, not less important is the consideration of artificial nets generated by some tailored methods. This approach allows one to extend the list with the nets that never occur in nature. The database EPINET mentioned above is the greatest project in generating ab initio nets. Studying occurrence of epinets, one can realize how stochastic is the realization of topological motifs in crystals. Therefore, since 
publishing the first EPINET release in 2005, the epinets were considered in any review mentioned above. Summarizing the results, we see that only 192 out of 14 532 epinets $(1.3 \%)$ have examples in nature; this fact proves the occurrence of nets to be strongly nonrandom. One more method for generating nets was developed by Blatov [16]; it is based on deriving all subnets for a particular net. Using this method for the RCSR nets, Blatov and Proserpio [16, 41] generated 9988 uninodal and 38304 binodal nets that were never described before; only for 92 and 100 of them, the crystal structure examples have so far been known. Resuming, the probability of a randomly taken topology to be realized in crystals is not larger than $1-2 \%$.

The current results on nets occurrence reveal general tendencies that require a theoretical interpretation: (i) there are a few nets that are much more frequent than others; (ii) diversity of single nets is much larger than of interpenetrating arrays; (iii) although bond type influences the occurrence of nets, some nets, like two-periodic sql, hcb, and fes or three-periodic srs, dia, and pcu, are most important for the substances of different nature; and (iv) the variety of nets directly depends on diversity of coordination centers; topology of nets with one kind of node only is the easiest to predict.

This analysis raises the questions to be crucial in the crystal structure prediction: (i) why frequent topologies are frequent and rare topologies are rare? (ii) Can any net type form an interpenetrating array, or some nets can occur single only? (iii) Can we predict topology for a given substance, with what probability? And what parameters should we know? Although these questions are not yet completely answered, there is an obvious progress in understanding as shown in detail in the next part.

\section{7}

\section{Some Properties of Nets Influencing the Crystal Structure}

\subsection{1}

\section{Symmetry of Nets and Embeddings}

The symmetry of a crystallographic periodic net can be described in terms of space groups; therefore, one can expect that the well-elaborated technique of mathematical crystallography can be used to derive some general properties of nets. Here we collect the results in this field with respect to prediction of the target motifs.

Theorem 1.1. The space group of a net is a subgroup of its automorphism group. This means that the symmetry owned by the net can be higher than the space-group symmetry of any of its embedding. If it is so, the net is noncrystallographic [7] and can have embeddings with space groups being not in a group-subgroup relation, for example, both in cubic and in hexagonal symmetry. Noncrystallographic nets always have symmetry operations that are inconsistent with a given space group. One can expect that these operations can appear as a kind of supersymmetry, and the substances built with such 
nets can have some special properties. However, nobody has yet studied the occurrence of noncrystallographic nets since there are no clear criteria to detect them in crystal structures.

Corollary 1.1. Site symmetry group of the net embedding is a subgroup of the symmetry group of a node in the net. This corollary restricts possible local topologies of structural units (i.e., both complex groups in the initial structure and the resulting coordination figures in the simplified net); for instance, the units with hexagonal symmetry are not suitable to realize in a cubic symmetry since they are forced to be distorted.

Corollary 1.2. The point group of a structural unit in the crystal must be a common subgroup of the point group of the corresponding node in the most symmetric embedding of the net and the point group of the isolated structural unit. A good illustration of this corollary is the consideration of possible topologies for molecular crystals. In particular, it follows that a uninodal net of a given topology may be realized in some space-group symmetry $G^{\prime}$ if, and only if, the index of $G^{\prime}$ in $G$ is a divisor of the order of the point group of the node in the most symmetric embedding of the net [49, 50]. This conclusion is proved by a comprehensive analysis of molecular packings [23, 26].

The theorem provides many useful conclusions to predict topological properties of the crystal architectures. In general, the specified topology restricts possible geometrical embeddings of the net, and vice versa. We can now answer the following crystallochemical questions [23, 26]:

1) Can a given topology be realized in a given space group? For instance, the diamond (dia) net cannot have hexagonal symmetry. Indeed, the automorphism group of dia is isomorphic to the space group $F d m$, but any hexagonal space group is not a subgroup of $F d \overline{3} m$ that contradicts Corollary 1.1. The same concerns the lonsdaleite (lon) net (so-called hexagonal diamond) that cannot have $F d \overline{3} m$ or any other cubic symmetry since its most symmetric embedding has the space group $\mathrm{PG}_{3} / \mathrm{mmc}$.

2) Which space groups can be realized for low-symmetric embeddings of a given topology? For this purpose, we have to find the most symmetric embedding of the net with the space group G; the space group of any other embedding of the net will be a subgroup of G. For example, both dia and lon can have embeddings with orthorhombic Pnna symmetry, that is, a common subgroup of $\mathrm{Fd} \overline{3} \mathrm{~m}$ and $\mathrm{PG}_{3} / \mathrm{mmc}$.

3) Can the coordination figure of a given symmetry be realized in a given embedding? For example, can we obtain dia with square-planar coordination? In the most symmetric embedding, any node of the diamond net has the symmetry $\overline{4} 3 \mathrm{~m}$ $\left(\mathrm{T}_{\mathrm{d}}\right)$. According to Corollary 1.1, we cannot obtain the regular square-planar coordination since the point group of the square $\left(4 / \mathrm{mmm}\right.$ or $\left.\mathrm{D}_{4 \mathrm{~h}}\right)$ is not a subgroup of $\overline{4} 3 \mathrm{~m}$. The same concerns rectangular coordination with the point group $m m m\left(\mathrm{D}_{2 \mathrm{~h}}\right)$. However, we can find some distorted planar coordination figures without inversion, describing by a subgroup of $\overline{4} 3 m$, say, $m m 2\left(\mathrm{C}_{2 \mathrm{v}}\right)$. 
4) Can a given structure unit occupy a particular Wyckoff position in a given net embedding? If we know the symmetry of the net embedding as well as the symmetry of a molecular group, we can use Corollary 1.2 to decide if the symmetries are compatible with each other. For example, molecules with inversion center prefer not to occupy the positions in the diamondoid nets because they have to lose the inversion center. The analysis of molecular packings $[23,26]$ proves this rule.

5) Will a given molecular packing in a given space symmetry be monomolecular or not? For instance, a uninodal diamond net is forbidden in the $P 2_{1}$ group because its index in $F d \overline{3} m, i=48$, is not a divisor of the order of the point group $\overline{4} 3 \mathrm{~m}$ of the node $(i=24)$, so at least two inequivalent nodes (molecular centers) should exist in the unit cell of a $P 2{ }_{1}$ diamond [23]; the total order of these nodes $(24 \times 2=48)$ is equal to $i$ and, hence, $i$ is its divisor that obeys Corollary 1.2.

In all the applications of the symmetry relations, the crucial is the space-group symmetry of the net in the most symmetric embedding. Once this symmetry has been found, the technique of mathematical crystallography can be in full applied to the net. Unfortunately, no tools were proposed to determine the automorphism group of the net except the geometrical approach based on the barycentric placement (Systre). Therefore, the nets that have a barycentric placement with collisions (including noncrystallographic nets) remain difficult to be considered within this approach. But in our experience, such nets are extremely rare, so Systre provide the most symmetric embedding for almost all chemically relevant examples.

Less strict, but not less important assumption concerning significance of high-symmetric nets for crystal chemistry was proposed in Ref. [21]. The authors revealed that most frequent underlying nets in MOFs are the nets with high space-group symmetry as well as high site symmetry of the nodes. It is noteworthy that the symmetry of the crystal can be and usually is low, but the symmetry of the underlying net itself ordinarily is high. A useful criterion of the high symmetry is transitivity. According to Ref. [21], the most important nets are regular, with transitivity 1111, that are srs, dia, nbo, pcu, and bcu. However, all nets with one kind of node and edge, that is, with transitivity 11 rs, are also suitable to be observed. This assumption explains why the most frequent nets in three-, four-, and six-coordinated MOFs are srs, dia, and pcu, respectively. The authors [21] substantiate the high-symmetry criteria by isotropy of the reacting system (melt or solution) and reaction centers (metal atoms). The reason could also be that the underlying net corresponds to some "primary" structure motif that determines the general method of ordering (the main modes in phonon spectra), while the details of interactions between structural units provide geometrical distortions of the structure and result in some subgroup of the space group of the underlying net. In any case, the physical reasons of this phenomenon require a more thorough consideration.

The role of high symmetry of the most symmetrical embedding of the net invokes some special classes of nets to be important for structure prediction [37]. 
1) Regular (transitivity 1111), quasiregular (transitivity 1112), and semiregular nets (transitivity 11rs) all have one kind of vertex and edge but differ by coordination figures that correspond to regular, quasiregular, or arbitrary convex polyhedra, respectively. All of them occur in crystal structures not occasionally [21, 37].

2) Edge-transitive nets have one kind of edge; in addition to the nets of class (1) the edge-transitive nets of transitivity 21 rs can be important for structures with two or more kinds of structural groups. Such edge-transitive nets as flu, pts, nia are frequent in MOFs $[21,51,52]$.

\subsection{2}

\section{Relations Between Nets}

Starting from a particular net, we can produce other nets that are unambiguously related to the initial one. Let us consider the types of such nets to be most important for materials science.

1) Subnets. One more important application of symmetry properties of periodic nets is the enumeration of all shortest ways to transform one net to another. For example, one can be curious of how to transform diamond to lonsdaleite by breaking and forming the minimum number of bonds. The space group of the intermediate net, supernet, or subnet must be a common subgroup of the space groups $F d \overline{3} m$ and $P G_{3} / m m c$; moreover this net must be a common supernet or subnet of dia and lon. A corresponding algorithm was proposed and realized in Ref. [16]. Using the program package TOPOS, one can construct a so-called net relation graph that indicates supernet-subnet relations for a given set of nets. With the net relation graph, we find that the pair dia-lon has two common three-coordinated uninodal subnets: utp (Pnna) and ths $\left(I 4_{1} /\right.$ amd $)$. Since Pnna is a common subgroup of $F d \overline{3} m$ and $P G_{3} / m m c$, utp can exist in its highest symmetry during the transition, while the possible symmetry ths is $C 2 / c$, that is, the maximal common subgroup of $F \overline{3} m$, $\mathrm{PG}_{3} / \mathrm{mmc}$, and $I 4_{1} /$ amd that retains ths uninodal. The number of pathways through a five-coordinated common supernet is much higher: according to Ref. [16], there are 93 such pathways. This information could be useful to predict structure transformations during phase transitions.

2) Dual nets. Since the nodes and edges of the dual net correspond to cages and rings of the initial net, the dual net can be considered as a system of void centers and channel lines between them. Anurova and Blatov [53] showed that dual nets can be efficiently used to analyze the migration paths in fast-ion conductors, to predict the existence and dimensionality of conductivity. In MOFs, the self-dual nets are of special interest [12] because, being combined with the initial net, they form strongly catenated network arrays. Thus, the uninodal self-dual nets srs, dia, cds, and pcu compose the main part (70\%) of interpenetrating MOF arrays [20]. Self-dual two-periodic nets can easily form a stacking, where nodes of one net are projected to the centers of rings of 

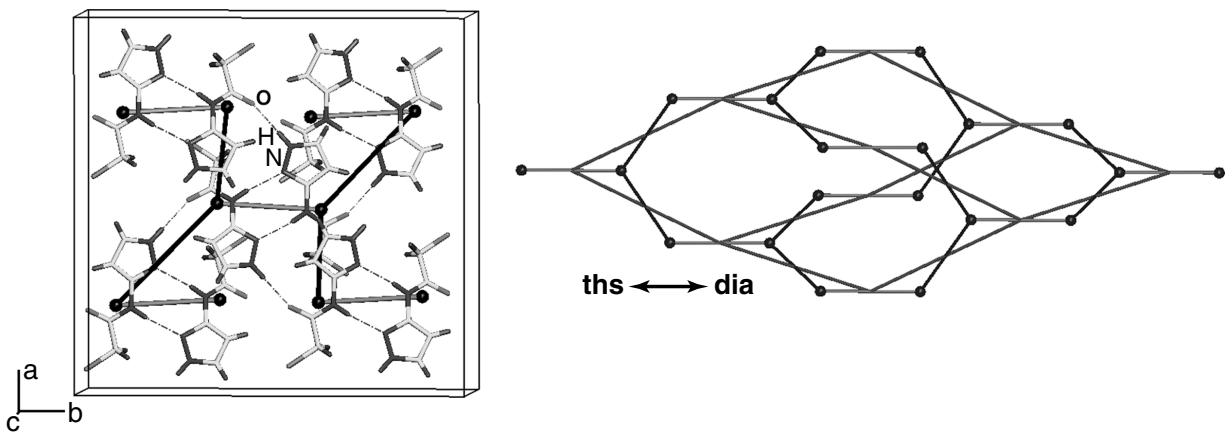

Figure 1.8 (a) The crystal structure of 3-(chloroacetamido)pyrazole with the ths underlying net and (b) corresponding partial edge net of the dia topology with the four-coordinates nodes on the middle of the gray edges. Molecular centroids and $\mathrm{H}$-bonded dimers $\left(R_{2}^{2}(8)\right.$ synthon $\left.[24,25]\right)$ are shown in the initial net as black balls and gray lines.

another net [54]. This feature provides existence of polytype series like the infinite series of close packings.

3) Edge nets. This type of nets can be useful for the design of molecular crystals. For instance, the net, whose nodes correspond to dimers, is an edge net with respect to the net of molecular centroids. Thus, both ths and srs nets have partial edge nets of the dia topology (Figure 1.8). Some other relations are given in Ref. [23].

4) Ring nets. A natural application of ring nets is the description of topology of coordination polymers with synthons. The ring net describing synthons is partial, as a rule [23]. For instance, an lvt net has partial ring net of the dia topology (Figure 1.9). This means that the synthons in the lvt net can arrange in a diamondoid motif. In turn, the lvt topology describes a partial ring net for the gis atomic net, and so on.

\subsection{3}

\section{Role of Geometrical and Coordination Parameters}

The criteria of high symmetry are not sufficient to explain an important role of some "default" nets. For example, the net sra has rather low symmetry (Imma), not minimal transitivity (33), does not belong to the special classes mentioned above, and its nodes are not allocated in the most symmetrical positions in the space group ( $8 i$ with site symmetry $m$ ). However, sra occupies the second place after dia in the list of the most frequent MOF topologies with tetrahedral units [21], while two nets following after it - sod $(\operatorname{Im} \overline{3} m)$ and qtz $\left(P_{6} 22\right)$ - have much higher symmetries and the smallest transitivities (12). Obviously, additional geometrical and chemical factors should manage the net occurrence.

The geometrical parameters are the property of the net embedding; therefore, special types of embeddings, like sphere packings, should be specified and explored. 

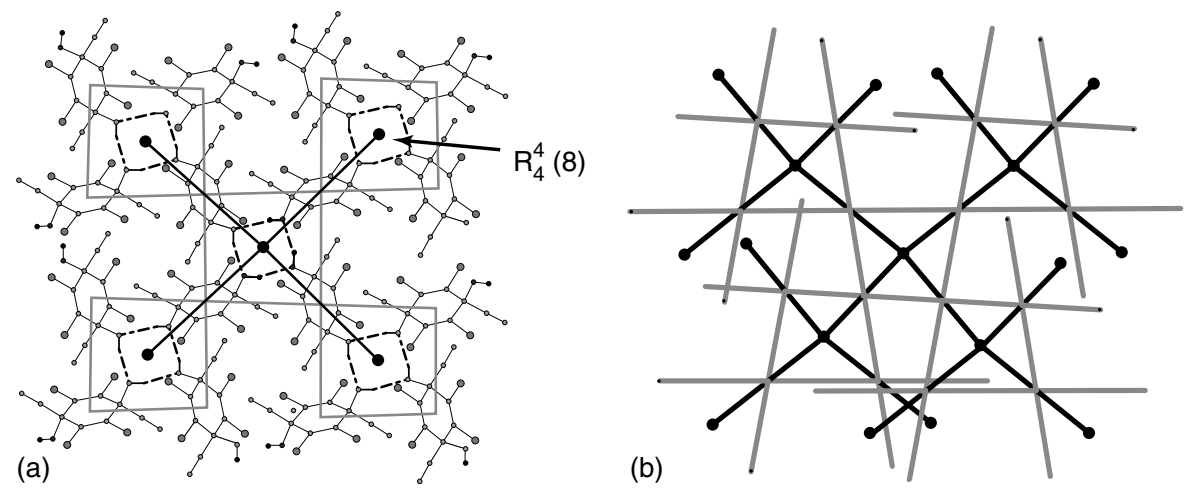

Figure 1.9 (a) The crystal structures of 2,3,5,6-tetrachlorotrans-1,4-diethynylcyclohexa-2,5-diene-1,4-diol (XEHKIE) with $\mathrm{R}_{4}^{4}$ (8) synthons; the initial net (gray lines) is formed by the centroids of molecules, while the partial ring net (black balls and lines) describes the synthons; (b) initial net lvt (gray) and partial ring net dia (black).

The importance of sphere packings reflects the importance of edge-transitive nets; however, in this case the equivalence of edges is not necessarily caused by symmetry reasons. It is a typical requirement that the links in the structure should be not the same, but of similar lengths (e.g., inorganic frameworks of oxofluorides or coordination polymers with chemically equivalent, but conformationally mobile ligands). It is noteworthy that all the nets mentioned above, including sra, are sphere packings. So if we have a compound with the ligands similar by length, the resulting net will be a sphere packing. Otherwise we can obtain a sphere packing or not; it depends on other reasons.

At present, not all sphere packings are derived even for uninodal nets. The occurrence of the known sphere packings is quite different; there are some of them that have no examples in crystal structures. In some cases, it can be explained by their low symmetry, but, in general, a deeper exploration of their geometrical properties is required. A more detailed classification of sphere packings accounting their ability to distortion due to degrees of freedom was proposed in Ref. [12]. However, no comprehensive study has yet been performed to check the occurrence of nets with respect to this classification.

The next important parameter is the form of coordination figure of the node. Not only distances (that are crucial for the concept of sphere packing) but also angles should be taken into account when anticipating the underlying net topology; moreover, in some cases there is a strong dependence between coordination figure and the underlying topology. The best correlations are observed for two-periodic nets. For instance, in the analysis of coordination polymers square-planar coordination leads to the sql topology almost in all cases, even if the rectangular is not regular. The same concerns triangular coordination figure that corresponds to the hcb underlying topology. We emphasize that hcb and sql are not unique topologies with planar three- or four-coordinated nodes, but they are the only uninodal 


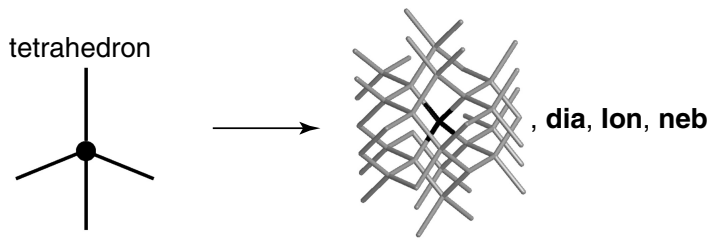

qtz
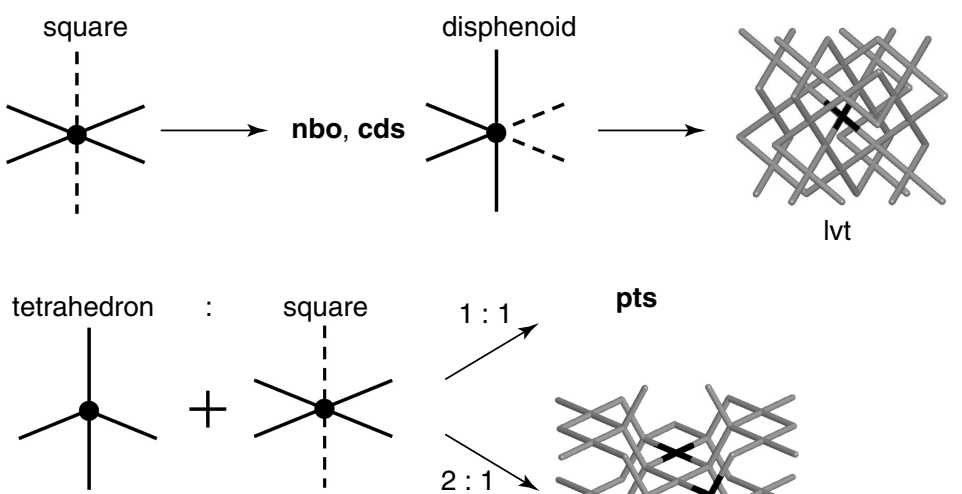

pts
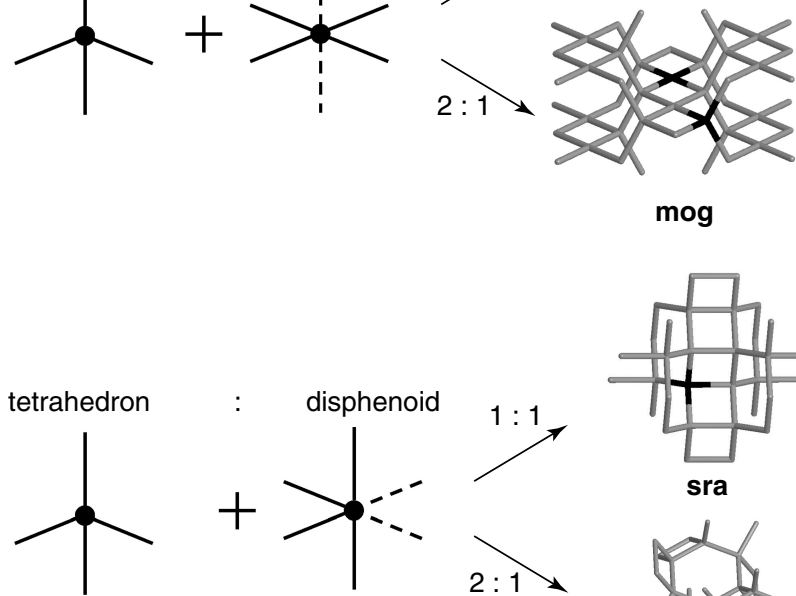

mog
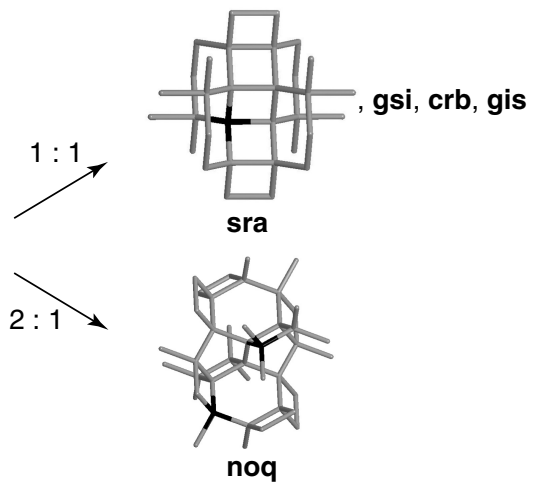

Figure 1.10 Tetrahedral, octahedral, and related complexes, coordination figures, and corresponding underlying nets in cyanides. Dashed lines correspond to links with terminal ligands. For some nets see Figure 1.3, for the others refer to the RCSR web page [29].

nets with triangular and square/rectangular coordination figures. Obviously, the highest possible plane-group symmetry plays a determinative role in this case. The required topology and geometry of the coordination figure can be designed by a proper introduction of terminal ligands into the coordination sphere of metal atom (Figure 1.10). 


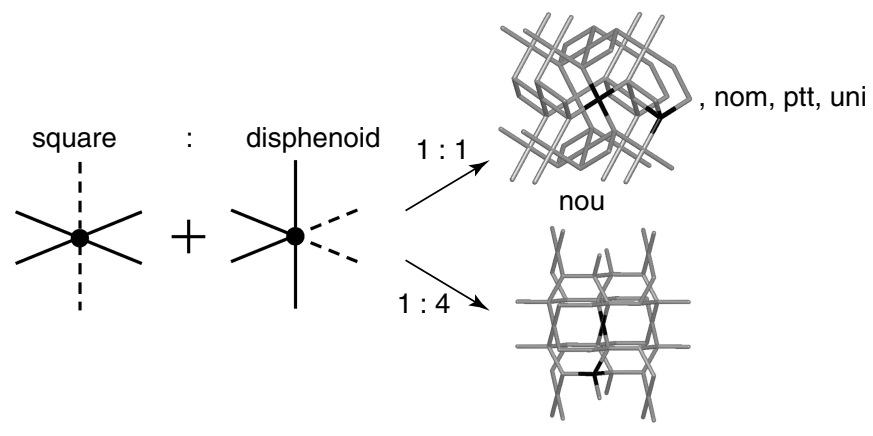

nor
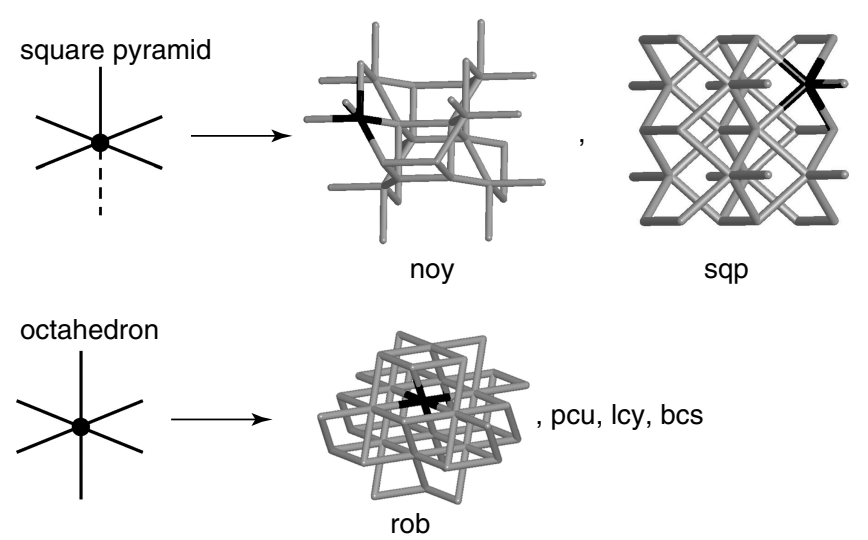

trigonal prism
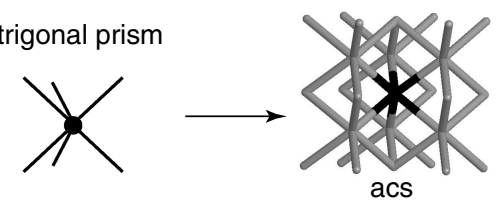

octahedron : trigonal prism

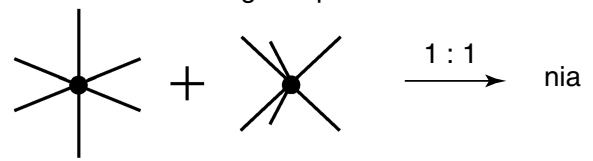

Figure 1.10 (continued.)

In the three-periodic coordination polymers, the correlation is not so strong; the same coordination figure can result in a diversity of underlying topologies [21]. However, if all ligands in the structure are similar by size, some special topologies of underlying net can be anticipated for any type of local coordination of metal atoms. For example, in cyanides there are the following correlations ([55], Figure 1.10):

- Four-coordinated nodes with tetrahedral coordination figure in almost all cases of cyanides result in dia topology; the only exception is lon topology, that is, 
very close to dia but is not edge transitive. One more edge-transitive topology with tetrahedral coordination, qtz, is realized only in inorganic cyanides. In this case, the spacers between tetrahedral centers are not cyanide ions, but linear groups $\left[\mathrm{Au}(\mathrm{CN})_{2}\right]^{-}$that, being more flexible provide less uniform quartz topology. More voluminous spacers, in particular, complex groups $\mathrm{Cu}$ (3,10-dipropyl-1,3,5,8,10,12-hexa-azacyclotetradecane) [56], lead to the neb topology, that is, locally very similar to dia [24].

- Four-coordinated nodes with square-planar coordination figure give two topologies, nbo and cds; the former one looks more preferable since it is edge-transitive (1111 vs. 1221).

- Four-coordinated nodes with disphenoidal coordination figure, which can be obtained from trigonal-bipyramidal coordination by removing one equatorial ligand or from octahedral coordination by removing two neighboring ligands, are realized in the mercury cyanide and correspond to the lvt topology. Obviously, the geometrical features of disphenoid (angles of $90^{\circ}$ and $180^{\circ}$ ) provide formation of four- and eight-rings that are typical for lvt, despite different coordination figure in lvt (square). We are not aware of the uninodal nets with disphenoidal coordination in the maximal symmetry embedding.

- Four-coordinated nodes with tetrahedral and square-planar coordination figures give rise to two topologies - pts or mog - depending on the nodes ratio 1:1 or 2:1, respectively.

- Four-coordinated nodes with tetrahedral and disphenoidal coordination figures lead to $\gamma$-Si (gsi), $\mathrm{CrB}_{4}$ (crb), $\mathrm{SrAl}_{2}$ (sra), or zeolite gismondine (gis) topologies at the nodes ratio $1: 1$. In this series, the number of four-rings meeting at the node increases from 0 to 3. Probably, different distortion of the disphenoidal coordination figures could manage the resulting underlying topology. At the nodes ratio 2:1, only the noq topology is realized.

- Four-coordinated nodes with square-planar and disphenoidal coordination figures emerge when there are two octahedral centers where two terminal ligands are allocated in trans- or cis-positions, respectively. At the ratio 1:1 the topologies nom, nou, ptt, or uni are observed, while the ratio 1:4 gives rise to the nor underlying net.

- Five-coordinated nodes with square-pyramidal coordination figure are derived from octahedral complexes with the single terminal ligand. This coordination produces the noy topology in all cases. If voluminous bridges like $\mathrm{Me}_{3} \mathrm{Sn}$ in $\left[\left(n-\mathrm{Bu}_{4} \mathrm{~N}\right)_{0.5}\left(\mathrm{Me}_{3} \mathrm{Sn}\right)_{3.5} \mathrm{Fe}(\mathrm{CN})_{6}\right][57]$ link the coordination centers, the sqp topology is realized.

- Six-coordinated nodes with octahedral coordination figure and short (cyanide) bridges always correspond to the pcu underlying net. The exceptions arise when the bridges are long or/and voluminous. Thus, in $\mathrm{N}(n-\mathrm{Bu})_{4}\left\{\mathrm{Ni}\left[\mathrm{Au}(\mathrm{CN})_{2}\right]_{3}\right\}$ [58], very rare bcs topology is realized. Although the net bcs is semiregular, it has strongly distorted coordination figure in the maximal symmetry embedding, since at the regular octahedral coordination two additional disconnected nodes are located nearby the central one (Figure 1.11a). In $\mathrm{N}(n-\mathrm{Bu})_{4}\left\{\mathrm{Ni}\left[\mathrm{Au}(\mathrm{CN})_{2}\right]_{3}\right\}$, this becomes possible owing to the long $\left[\mathrm{Au}(\mathrm{CN})_{2}\right]$ bridges; out of eight $\mathrm{Ni}$ atoms surrounding 


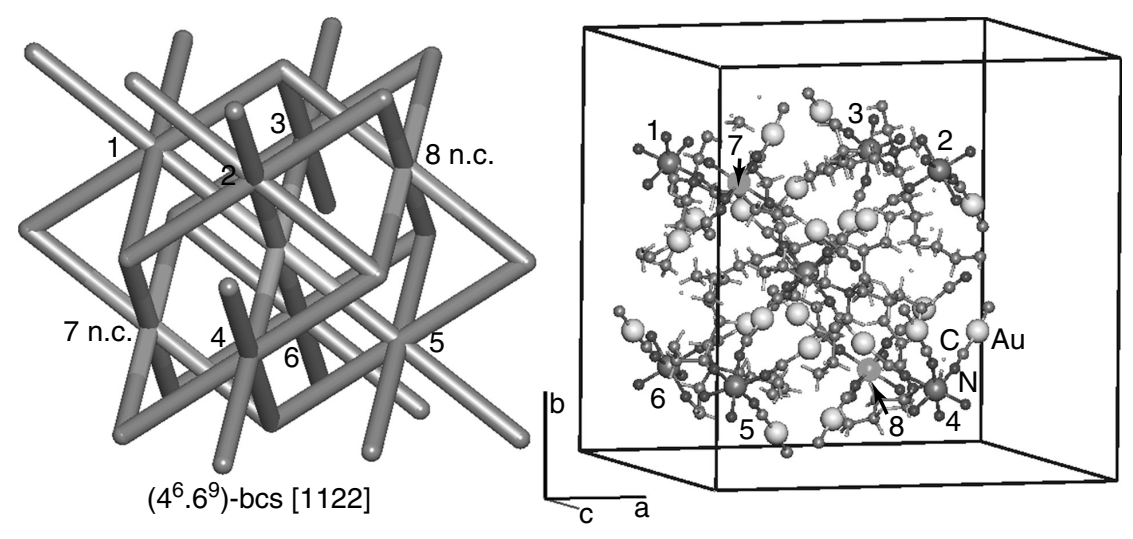

Figure 1.11 (a) Idealized bcs net with the six coordinates nodes $(1-6)$ and the two close but not directly connected to the central one (7,8 n.c.); (b) a fragment of the $\mathrm{N}(\mathrm{n}-\mathrm{Bu})_{4}\left\{\mathrm{Ni}\left[\mathrm{Au}(\mathrm{CN})_{2}\right]_{3}\right\}$ structure. $\mathrm{Ni}$ atoms $(1-8)$ form a cube around the central $\mathrm{Ni}$. The two atoms disconnected to the central one $(7,8)$ are marked by arrows.

the central $\mathrm{Ni}$ at distances $10.1-10.2 \AA$, only six are connected to it via $\left[\mathrm{Au}(\mathrm{CN})_{2}\right]$ groups (Figure 1.11b). In isomorphic pair $\left\{(n-\mathrm{Bu})_{3} \mathrm{Sn}\right\}_{3}\left[\mathrm{M}(\mathrm{CN})_{6}\right](\mathrm{M}=\mathrm{Fe}, \mathrm{Co})$ [59], the three-rings required for emerging the lcy topology are formed of voluminous $\left[(n-\mathrm{Bu})_{3} \mathrm{Sn}\right]$ bridges. If the coordination center is extended with a binuclear cluster like $\left[\mathrm{Cu}_{2}(\mathrm{CN})_{2}\right]$ in $\left[\mathrm{Cu}_{5}(\mathrm{CN})_{6}(\mathrm{DMF})_{4}\right][60]$ or $\left[\mathrm{Cu}_{4} \mathrm{Zn}(\mathrm{CN})_{6}(\mathrm{DMF})_{4}\right]$ [61], another topology, rob, is realized.

- Six-coordinated nodes with trigonal-prismatic coordination figure occur only in $\mathrm{Eu}\left[\mathrm{Ag}(\mathrm{CN})_{2}\right]_{3}\left(\mathrm{H}_{2} \mathrm{O}\right)_{3}$ where three terminal water molecules extend the Eu coordination number up to typical $(\mathrm{CN}=9)$. The underlying topology is acs.

- Six-coordinated nodes with octahedral and trigonal-prismatic coordination figures occur only in the ratio $1: 1$ and in all cases give rise to the nia underlying net.

This list of correspondences can be extended with the nets with different degrees of nodes (e.g., four-, six-coordinated nets with tetrahedral and octahedral coordination figures in the ratio $1: 2$ result in the fsh topology), and the given data lead to similar conclusions as for two-periodic coordination polymers: the coordination figure strongly determines the underlying topology. Almost in all the cases, the shape of coordination polyhedron (considered without terminal ligands) coincides with the coordination figure of corresponding node in the most symmetrical embedding of the underlying net. The correspondences show that variation of the number and positions of the terminal ligands allows one to design the required underlying topology with a high probability. The cases with several possible underlying topologies (like nom, nou, ptt, or uni) 
have to be separately studied. Obviously, the shape, size, and number of terminal ligands as well as solvate molecules should be taken into account. The structure of the coordination center can also influence the underlying topology. Taking together all the factors could allow us to predict the 3D motifs more rigorously. We see that all these parameters sufficiently expand more general symmetry criteria. In more complicated structures containing different bridge ligands of different composition, the correlations could be more subtle, but they could be somehow catalogued. This is an important task of crystal design.

Similar regularities control crystal structures of other nature. Thus, Baburin and Blatov [23] showed that the topology of 3D frameworks of hydrogen-bonded molecules depends on the local arrangement of the molecule. They showed that strongly distorted tetrahedral environment of the molecule results in qtz or dmp topologies, while the square-like coordination leads to cds or lvt underlying topologies. Baburin [26] analyzed the influence of arrangement of active centers of hydrogen bonding over the molecule surface on the topology of molecular packings in coordination compounds. For instance, octahedral complexes with six ligands containing functional groups involved into hydrogen bonding prefer to form the pcu motif.

The underlying topology can be predetermined even more precisely if one extends the coordination figure to some larger part of the structure framework, that is, inherent to the resulting simplified net. This part can be referred to as a secondary building unit (SBU) of the net. To predict the net topology with a particular SBU, one has to know how specific is this SBU, and to what number of different nets it corresponds. For example, using the adamantane-like units one can construct the dia underlying net with much greater probability than starting from tetrahedral complexes. This approach lies in the base of reticular chemistry and was successfully used to design and synthesize new MOF materials $[21,30,62]$.

In summary, the chemical factors influencing the local geometric and topological properties of the structure determine the underlying net topology according to the five-level scheme: coordination number-complex group-coordination figure - secondary building unit-underlying topology (Figure 1.12). If the ligands are either monodentate (terminal) or rather short bidentate-bridge, the scheme can be easily interpreted. The coordination number of the complexing atom or a cluster predetermines the maximum number of connected ligands in the complex group and, hence, the maximum number of nodes in the coordination figure; within this number, the coordination figure can be modified by introducing terminal ligands. The same coordination figure can correspond to different complex groups where the ligands vary by composition, shape, and size. At last, the same coordination figure can be built in different SBUs that provide different underlying topologies, thanks to various interstitial species or thermodynamic conditions [46, 47]. Thus, there is some diversity in final topology of the underlying net, but, as was shown above, this diversity is not so vast. 


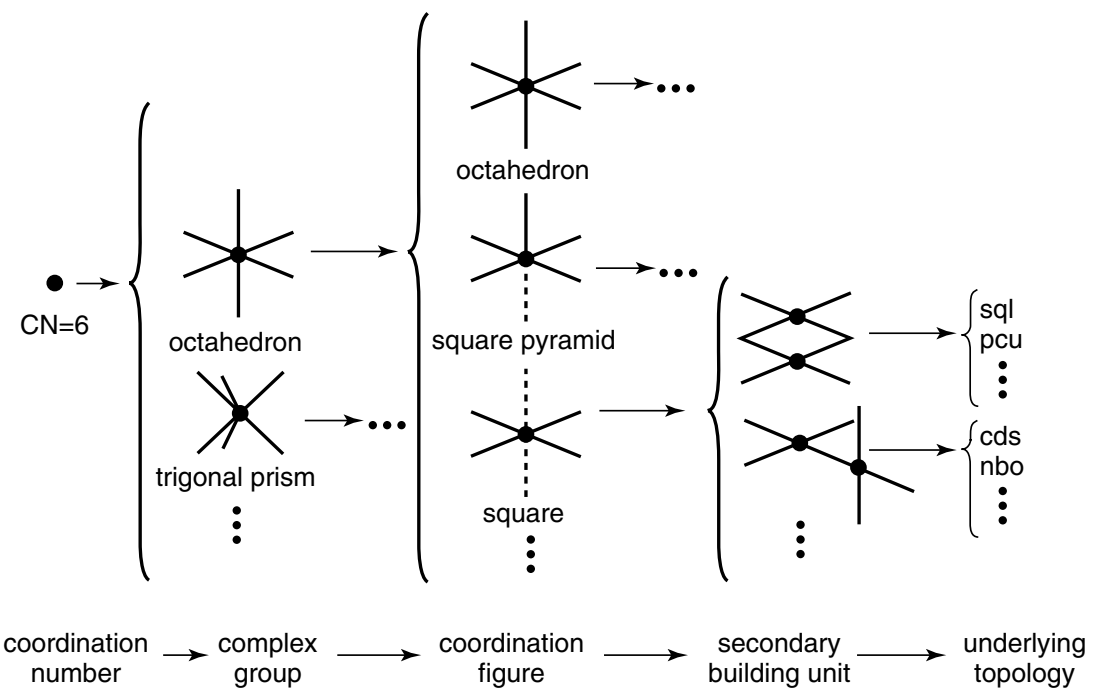

Figure 1.12 An example of the five-level hierarchical scheme of the underlying topology prediction starting from a complexing atom with coordination number 6 , for example, $\mathrm{Fe}, \mathrm{Co}$, or $\mathrm{Ni}$.

\section{8}

\section{Outlook}

The investigations of previous years mainly answered Wells' question - indeed, only rather small set of nets frequently occurs in crystal structures and we know almost all nets from this set. However, a number of new challenges emerged that will determine the development of periodic-graph approaches in the near future. We see the following main problems that should be solved at first: (i) it should become a standard for any structure investigation to provide the information on the underlying topology of possible structure representations along with crystallographic data; (ii) crystallographic databases should be integrated with the crystallochemical databases; (iii) the crystallochemical databases should be completed with all 2D and 3D representations of all known crystal structures that will enable the user to find similar topological motifs in different structures and to foresee possible topologies in new compounds by looking for chemical and structural prototypes in the databases; (iv) a database should be created that will collect strict interrelations between different topological representations like the interrelations "net-dual net," "net-edge net," "net-partial ring net," and so on. to let the user know in what structure representation he can get the required net topology; (v) the known parameters that influence the underlying topology (Section 1.7) should be deeper explored and new ones have to be discovered; (vi) an expert system should be developed that will use all the above-mentioned correlations and predict the structural dependencies in the form "complexing atom 
+ ligands $\rightarrow$ list of possible complex groups $\rightarrow$ list of possible SBUs $\rightarrow$ list of underlying topologies" with occurrence probabilities and allowable space-group symmetries. Successful solution of these problems will inspire further progress in materials science and crystal chemistry.

\section{References}

1. Öhrström, L. and Larsson, K. (2005) Molecule-based Materials: The Structural Network Approach, Elsevier, Amsterdam.

2. Carlucci, L., Ciani, G., and Proserpio, D.M. (2007) in Making Crystals by Design - Methods Techniques and Applications (eds D. Braga and G. Grepioni), Wiley-VCH Verlag $\mathrm{GmbH}$, Weinheim, pp. 58-85.

3. Wells, A.F. (1977) Three-Dimensional Nets and Polyhedra, Interscience, New York.

4. Chung, S.J., Hahn, Th., and Klee, W.E. (1984) Nomenclature and generation of three-periodic nets: the vector method. Acta Cryst., A40, 42-50.

5. Klein, H.-J. (1996) Systematic generation of models for crystal structures. Math. Model. Sci. Comput., 6, 325-330.

6. Delgado-Friedrichs, O. and O'Keeffe, M. (2003) Identification of and symmetry computation for crystal nets. Acta Cryst., A59, 351-360.

7. Klee, W.E. (2004) Crystallographic nets and their quotient graphs. Cryst. Res. Technol., 39, 959-968.

8. Eon, J.-G. (2005) Graph-theoretical characterization of periodicity in crystallographic nets and other infinite graphs. Acta Cryst., A61, 501-511.

9. Thimm, G. (2009) Crystal topologies: the achievable and inevitable symmetries.

Acta Cryst., A65, 213-226.

10. Goetzke, K., Klein, J.-H., and Kandzia, P. (1988) in Graph-Theoretic Concepts in Computer Science, Proceedings of the International Workshop WG '87, June 29 - July 1, 1987, Kloster Banz/Staffelstein, FRG (eds H. Göttler and H.J. Schneider), Springer-Verlag, Berlin, pp. 242-254.

11. Koch, E., Fischer, W., and Sowa, H. (2006) Interpenetration of homogeneous sphere packings and of two-periodic layers of spheres. Acta Cryst., A62, 152-167.

12. Delgado-Friedrichs, O., Foster, M.D., O'Keeffe, M., Proserpio, D.M., Treacy, M.M.J., and Yaghi, O.M. (2005) What do we know about three-periodic nets? J. Solid State Chem., 178, 2533-2554.

13. Brunner, G.O. and Laves, F. (1971) Zum problem der koordinationszahl. Wiss. Z. Techn. Univ. Dres., 20, 387-390.

14. Goetzke, K. and Klein, J.-H. (1991) Properties and efficient algorithmic determination of different classes of rings in finite and infinite polyhedral networks. J. Non-Cryst. Solids, 127, 215-220.

15. O'Keeffe, M. and Hyde, S.T. (1997) Vertex symbols for zeolite nets. Zeolites, 19, 370-374.

16. Blatov, V.A. (2007) Topological relations between three-dimensional periodic nets. I. Uninodal nets. Acta Cryst., A63, 329-343.

17. Fischer, W. and Koch, E. (1989) Genera of minimal balance surfaces. Acta Cryst., A45, 726-732.

18. Delgado-Friedrichs, O., O’Keeffe, M., and Yaghi, O.M. (2003) Three-periodic nets and tilings: regular and quasiregular nets. Acta Cryst., A59, 22-27.

19. Hyde, S.T., Delgado Friedrichs, O., Ramsden, S.J., and Robins, V. (2006) Towards enumeration of crystalline frameworks: The 2D hyperbolic approach. Solid State Sci., 8, 740-752.

20. Blatov, V.A., Carlucci, L., Ciani, G., and Proserpio, D.M. (2004) Interpenetrating metal-organic and inorganic 3D networks: a computer-aided systematic investigation. Part I. Analysis of the Cambridge structural database. CrystEngComm., 6, 377-395.

21. Ockwig, N.W., Delgado-Friedrichs, O., O'Keeffe, M., and Yaghi, O.M. (2005) Reticular chemistry: occurrence and taxonomy of nets and grammar for the 
design of frameworks. Acc. Chem. Res., 38, 176-182.

22. Baburin, I.A., Blatov, V.A., Carlucci, L., Ciani, G., and Proserpio, D.M. (2005) Interpenetrating metal-organic and inorganic 3D networks: a computer-aided systematic investigation. Part II. Analysis of the inorganic crystal structure database (ICSD). J. Solid State Chem., 178, 2452-2474.

23. Baburin, I.A. and Blatov, V.A. (2007) Three-dimensional hydrogen-bonded frameworks in organic crystals: a topological study. Acta Cryst., B63, 791-802.

24. Baburin, I.A., Blatov, V.A., Carlucci, L., Ciani, G., and Proserpio, D.M. (2008) Interpenetrated 3D networks of H-bonded organic species: a systematic analysis of the Cambridge structural database. Cryst. Growth Des., 8 , 519-539.

25. Baburin, I.A., Blatov, V.A., Carlucci, L., Ciani, G., and Proserpio, D.M. (2008) Interpenetrated three-dimensional hydrogen-bonded networks from metal-organic molecular and one- or two-dimensional polymeric motifs. CrystEngComm., 10, 1822-1838.

26. Baburin, I.A. (2008) Hydrogen-bonded frameworks in molecular metal-organic crystals: the network approach. $Z$. Kristallogr., 223, 371-381.

27. Blatov, V.A. (2006) Multipurpose crystallochemical analysis with the program package TOPOS. IUCr Comp. Commun. Newsl., 7, 4-38.

28. Blatov, V.A., Delgado-Friedrichs, O., O'Keeffe, M., and Proserpio, D.M. (2007) Three-periodic nets and tilings: natural tilings for nets. Acta Cryst., A63, 418-425.

29. O'Keeffe, M., Peskov, M.A., Ramsden, S.J., and Yaghi, O.M. (2008) The reticular chemistry structure resource (RCSR) database of, and symbols for, crystal nets. Acc. Chem. Res., 41, 1782-1789.

30. Yaghi, O.M., O’Keeffe, M., Ockwig, N.W., Chae, H.K., Eddaoudi, M., and Kim, J. (2003) Reticular synthesis and the design of new materials. Nature, 423, 705-714.
31. Delgado-Friedrichs, O. and O'Keeffe, M. (2005) Crystal nets as graphs: terminology and definitions. J. Solid State Chem., 178, 2480-2485.

32. Francl, M. (2009) Stretching topology. Nat. Chem., 1, 334-335.

33. Proserpio, D.M. (2010) Polycatenation weaves a 3D web. Nat. Chem., 2, 435-436.

34. Koch, E. and Fischer, W. (1978) Types of sphere packings for crystallographic point groups, rod groups and layer groups. Z. Kristallogr., 148, 107-152.

35. Thimm, G. and Klee, W.E. (1997) Zeolite cycle sequences. Zeolites, 19, 422-424.

36. O'Keeffe, M. and Hyde, B.G. (1996) Crystal Structures. I. Patterns and Symmetry, Mineralogical Society of America, Washington, DC.

37. Delgado-Friedrichs, O., O'Keeffe, M., and Yaghi, O.M. (2007) Taxonomy of periodic nets and the design of materials. Phys. Chem. Chem. Phys., 9, 1035-1043.

38. Bonneau, C., Delgado Friedrichs, O., O'Keeffe, M., and Yaghi, O.M. (2004) Three-periodic nets and tilings: minimal nets. Acta Cryst., A60, 517-520.

39. Sowa, H., Koch, E., and Fischer, W. (2007) Orthorhombic sphere packings. II. Bivariant lattice complexes. Acta Cryst., A63, 354-364.

40. Sowa, H. (2009) Three new types of interpenetrating sphere packings. Acta Cryst., A65, 326-327.

41. Blatov, V.A. and Proserpio, D.M. (2009) Topological relations between three-periodic nets. II. Binodal nets. Acta Cryst., A65, 202-212.

42. Ramsden, S.J., Robins, V., and Hyde, S.T. (2009) Three-dimensional Euclidean nets from two-dimensional hyperbolic tilings: kaleidoscopic examples. Acta Cryst., A65, 81-108.

43. Blatov, V.A. (2006) A method for hierarchical comparative analysis of crystal structures. Acta Cryst., A62, 356-364.

44. O'Keeffe, M., Eddaoudi, M., Li, H., Reineke, T., and Yaghi, O.M. (2000) Frameworks for extended solids: geometrical design principles. J. Solid State Chem., 152, 3-20.

45. Dolomanov, O.V., Bourhis, L.J., Gildea, R.J., Howard, J.A.K., and Puschmann, 
H. (2009) OLEX2: a complete structure solution, refinement and analysis program. J. Appl. Cryst., 42, 339-341.

46. Baburin, I.A., Leoni, S., and Seifert, G. (2008) Enumeration of not-yet-synthesized zeolitic zinc imidazolate MOF networks: a topological and DFT approach. J. Phys. Chem. B, 112, 9437-9443.

47. Lewis, D.W., Ruiz-Salvador, A.R., Gómez, A., Rodriguez-Albelo, L.M., Coudert, F.-X., Slater, B., Cheetham, A.K., and Mellot-Draznieks, C. (2009) Zeolitic imidazole frameworks: structural and energetics trends compared with their zeolite analogues. CrystEngComm., 11, 2272-2276.

48. Blatov, V.A. and Peskov, M.V. (2006) A comparative crystallochemical analysis of binary compounds and simple anhydrous salts containing pyramidal anions $\mathrm{LO}_{3}(\mathrm{~L}=\mathrm{S}, \mathrm{Se}, \mathrm{Te}, \mathrm{Cl}, \mathrm{Br}, \mathrm{I})$. Acta Cryst., B62, 457-466.

49. Klee, W.E. (1974) Al/Si distributions in tectosilicates: a graph-theoretical approach. Z. Kristallogr., 140, 154-162.

50. Fischer, W. and Koch, E. (1978) Limiting forms and comprehensive complexes for crystallographic point groups, rod groups and layer groups. Z. Kristallogr., 147, 255-273.

51. Delgado-Friedrichs, O., O'Keeffe, M., and Yaghi, O.M. (2006) Three-periodic nets and tilings: edge-transitive binodal structures. Acta Cryst., A62, 350-355.

52. Delgado-Friedrichs, O. and O'Keeffe, M. (2007) Three-periodic tilings and nets: face-transitive tilings and edge-transitive nets revisited. Acta Cryst., A63, 344-347.

53. Anurova, N.A. and Blatov, V.A. (2009) Analysis of ion-migration paths in inorganic frameworks by means of tilings and Voronoi-Dirichlet partition: a comparison. Acta Cryst., B65, 426-434.

54. O'Keeffe, M. (1992) Self-dual plane nets in crystal chemistry. Aust. J. Chem., 45, 1489-1498.
55. Virovets, A.V., Blatov, V.A., and Peresypkina, E.V., unpublished results.

56. Yuan, A.-H., Zhou, H., Chen, Y.-Y., and Shen, X.-P. (2007) A novel three-dimensional $\mathrm{Cu}(\mathrm{II})-\mathrm{Mo}(\mathrm{IV})$ bimetallic complex: synthesis, crystal structure, and magnetic properties. $J$. Mol. Struct., 826, 165-169.

57. Schwarz, P., Eller, S., Siebel, E., Soliman, T.M., Fischer, R.D., Apperley, D.C., Davies, N.A., and Harris, R.K. (1996) Template-driven syntheses of polymeric metal cyanides: a chiral nanoporous host for the $n \mathrm{Bu}_{4} \mathrm{~N}^{+}$ion. Angew. Chem. Int. Ed., 35, 1525-1527.

58. Lefebvre, J., Chartrand, D., and Leznoff, D.B. (2007) Synthesis, structure and magnetic properties of 2-D and 3-D [cation] $\left\{\mathrm{M}\left[\mathrm{Au}(\mathrm{CN})_{2}\right]_{3}\right\}(\mathrm{M}=\mathrm{Ni}, \mathrm{Co}) \mathrm{co}-$ ordination polymers. Polyhedron, 26, 2189-2199.

59. Niu, T., Lu, J., Wang, X., Korp, J.D., and Jacobson, A.J. (1998) Syntheses and structural characterizations of two three-dimensional polymers: $\left[\left\{\left(n-\mathrm{C}_{4} \mathrm{H}_{9}\right)_{3} \mathrm{Sn}\right\}_{3} \mathrm{M}(\mathrm{CN})_{6}\right](\mathrm{M}=\mathrm{Fe}, \mathrm{Co})$. Inorg. Chem., 37, 5324-5328.

60. Peng, S.-M. and Liaw, D.-S. (1986) $\mathrm{Cu}$ (II) ion catalytic oxidation of o-phenylenediamine and diaminomaleonitrile and the crystal structure of the final products $\left(\mathrm{C}_{12} \mathrm{~N}_{4} \mathrm{H}_{11}\right)\left(\mathrm{ClO}_{4}\right) \mathrm{H}_{2} \mathrm{O}$ and $\left[\mathrm{Cu}_{5}(\mathrm{CN})_{6}(\mathrm{dmf})_{4}\right]$. Inorg. Chim. Acta, 113, L11-L12.

61. Cui, C.-P., Lin, P., Du, W.-X., Wu, L.-M., Fu, Z.-Y., Dai, J.-C., Hu, S.-M., and Wu, X.-T. (2001) Synthesis and structures of two novel heterometallic polymers. Inorg. Chem. Commun., 4, 444-446.

62. Eddaoudi, M., Moler, D.B., Li, H., Chen, B., Reineke, T.M., O’Keeffe, M., and Yaghi, O.M. (2001) Modular chemistry: secondary building units as a basis for the design of highly porous and robust metal-organic carboxylate frameworks. Acc. Chem. Res., 34, 319-330. 